\title{
Morbid obesity and metabolic syndrome in Ossabaw miniature swine are associated with increased platelet reactivity
}

This article was published in the following Dove Press journal: Diabetes, Metabolic Syndrome and Obesity:Targets and Therapy I4 March 20II

Number of times this article has been viewed

\author{
Rolf P Kreutz ${ }^{1,2}$ \\ Mouhamad Alloosh ${ }^{3}$ \\ Khaled Mansour' \\ Zachary Neeb ${ }^{3}$ \\ Yvonne Kreutz ${ }^{2}$ \\ David A Flockhart ${ }^{2}$ \\ Michael Sturek ${ }^{3}$ \\ 'Krannert Institute of Cardiology, \\ ${ }^{2}$ Division of Clinical Pharmacology, \\ ${ }^{3}$ Department of Cellular and \\ Integrative Physiology, Indiana \\ University School of Medicine, \\ IN, USA
}

Background: Metabolic syndrome (MetS) and type 2 diabetes mellitus in humans are associated with increased platelet activation and hyperreactivity of platelets to various agonists. Ossabaw swine develop all the hallmarks of MetS including obesity, insulin resistance, hypertension, dyslipidemia, endothelial dysfunction, and coronary artery disease when being fed excess calorie atherogenic diet. We hypothesized that Ossabaw swine with MetS would exhibit increased platelet reactivity compared with lean pigs without MetS.

Materials and methods: Ossabaw swine were fed high caloric, atherogenic diet for 44 weeks to induce MetS $(n=10)$ and were compared with lean controls without MetS that had been fed normal calorie standard diet $(n=10)$. Light transmittance aggregometry was performed using adenosine diphosphate (ADP), collagen, thrombin, and arachidonic acid (AA) at different concentrations. Dose response curves and EC50 were calculated. Glucose tolerance testing and intravascular ultrasound study of coronary arteries were performed.

Results: MetS pigs compared with lean controls were morbidly obese, showed evidence of arterial hypertension, elevated cholesterol, low-density lipoprotein/high-density lipoprotein, and triglycerides, and insulin resistance. Platelets from MetS pigs were more sensitive to ADPinduced platelet aggregation than leans (EC50: $1.83 \pm 1.3 \mu \mathrm{M}$ vs $3.64 \pm 2.2 \mu \mathrm{M} ; P=0.02$ ). MetS pigs demonstrated higher platelet aggregation in response to collagen than lean pigs (area under the curve: $286 \pm 74$ vs $198 \pm 123 ; P=0.037$ ) and a trend for heightened response to AA (AUC: $260 \pm 151$ vs $178 \pm 145 ; P=0.13$ ). No significant difference was found for platelet aggregation in response to thrombin.

Conclusions: MetS in Ossabaw swine is associated with increased reactivity of platelets to ADP and collagen. The Ossabaw swine may be a practical, large animal model for the study of certain aspects of platelet pathophysiology and examine vascular devices in a metabolic environment comparable to humans with MetS.

Keywords: coronary artery disease, insulin resistance, glucose intolerance, platelet

\section{Introduction}

The term metabolic syndrome (MetS) has been used to describe the combination and co-occurrence of abdominal obesity, insulin resistance, impaired glucose tolerance, hypertension, and dyslipidemia (increased low-density lipoprotein/high-density lipoprotein [LDL/HDL] and triglycerides). These cardiovascular risk factors interact with each other and accelerate the progression of atherosclerosis and arterial thrombosis. ${ }^{1-3}$ Although MetS is considered to represent heterogeneous traits, insulin resistance and glucose intolerance associated with obesity are considered to be central to the pathophysiology. MetS depends on genetic predisposition, as well as environmental conditions that allow its progression. An abundant high-calorie, high-fat diet, and
Correspondence: Rolf P Kreutz

Krannert Institute of Cardiology, Indiana University School of Medicine, 1800 N.Capitol Ave, ME-400, Indianapolis, IN 46202, USA

$\mathrm{Tel}+\mathrm{I} 3179620561$

$\mathrm{Fax}+13179620113$

Email rkreutz@iupui.edu 
sedentary lifestyle are considered central to the development of MetS in individuals at risk. Estimates suggest that approximately $24 \%$ of Americans suffer from MetS as defined by Adult Treatment Panel III (ATP III) guidelines. ${ }^{4}$ The onset of MetS and further progression to type 2 diabetes is associated with findings of endothelial dysfunction, increased platelet activation, and platelet hyperreactivity. ${ }^{1,5-9}$

The Ossabaw miniature swine has recently been identified as a large animal model for the study of the pathogenesis of MetS. ${ }^{10,11}$ Ossabaw swine, from Ossabaw Island off the coast of Georgia, exhibit a thrifty genotype, which allows them to store large amounts of fat for survival during seasonal famine accentuated by the isolated location of the island. When fed an excess calorie atherogenic diet over several months, Ossabaw swine develop all the pathological aspects of MetS, including hypertension, dyslipidemia, glucose intolerance, endothelial dysfunction, steatohepatitis, as well as atherosclerosis in the coronary vasculature. ${ }^{10-20}$

So far no study has examined the effects of MetS in Ossabaw swine on platelet aggregation. We hypothesized that Ossabaw swine with MetS exhibit a pro-aggregatory phenotype evidenced by increased reactivity of platelets to agonists compared with lean pigs without MetS.

\section{Materials and methods}

All experimental procedures involving animals were approved by the Indiana University Animal Care and Use Committee and complied fully with the Guide for the Care and Use of Laboratory Animals and the American Veterinary Medical Association Panel on Euthanasia. ${ }^{21,22}$ For all surgical procedures and euthanasia, anesthesia was induced by tiletamine-zolazepam $(5 \mathrm{mg} / \mathrm{kg})$ and xylazine $(2.2 \mathrm{mg} / \mathrm{kg})$ given intramuscularly; anesthesia was maintained with isoflurane gas $(<4 \%)$. Pigs were euthanized by cardiectomy while anesthetized.

\section{Metabolic syndrome pig model}

Ossabaw miniature swine (Sus scrofa) were provided from the colony in the Comparative Medicine Program of Indiana University School of Medicine and Purdue University. Swine aged 6 to 7 months at the beginning of the study were fed for 44 weeks either a lean standard chow of $2400 \mathrm{kCal} /$ day containing $8 \%$ fat (lean control group, $\mathrm{n}=10$ ) or an excess calorie atherogenic diet of $6000 \mathrm{kcal} /$ day containing 45\% fat (MetS group, $\mathrm{n}=10) .{ }^{17}$ The details of this pig model, including the composition of the atherogenic diet (5B4L; Purina TestDiet, Richmond, IN) and lean standard chows (5L80; Purina TestDiet) have been published previously. ${ }^{17}$ In this model, total cholesterol increased about 5-fold and triglycerides about 3-fold.
Body weights were measured on a weekly basis in all animals. Blood pressure was measured with a tail cuff sphygmomanometer in conscious swine in a low-stress restraint sling. ${ }^{10,11}$

\section{Intravenous glucose tolerance test}

Swine were acclimatized to low-stress restraint in a specialized sling for 5 to 7 days before the intravenous glucose tolerance test (IVGTT). ${ }^{10,11}$ Swine were then fasted overnight and after inducing anesthesia, the right jugular vein was catheterized percutaneously. ${ }^{10,11}$ As previously described and after collecting fasting blood samples, pigs were administered an intravenous bolus of $1 \mathrm{~g}$ glucose $/ \mathrm{kg}$ body weight, and blood samples were obtained at 5, 10, 20,30, 40, 50, and 60 minutes after injection. ${ }^{10,11}$ Blood glucose was measured using a YSI 2300 Stat Plus analyzer (YSI, Yellowsprings, OH). Plasma insulin levels were determined as previously described. ${ }^{11}$ The area under the curve (AUC) was calculated with the baseline at 0 .

\section{Plasma lipids}

Fasting plasma samples obtained the day of the IVGTT from the jugular catheter were analyzed for triglycerides, total cholesterol and HDL fraction by using a standard enzymatic kit (Thermo Trace, Melbourne, Australia) as previously described. ${ }^{11}$ LDL was calculated using the Friedewald equation $(\mathrm{LDL}=$ total cholesterol $-\mathrm{HDL}-($ triglycerides $/ 5))$.

\section{Intravascular ultrasound}

Intravascular ultrasound (IVUS) study of both left anterior descending and circumflex coronary arteries using a 30 or $40 \mathrm{MHz}$ IVUS imaging catheter (Ultracross 3.2 or Discovery; Boston Scientific, Natick, MA) was performed as previously described. ${ }^{10-13,15,16,18}$ Atheroma was defined as the presence of soft or fibrous plaque adjacent to the lumen and separated from the adventitia by an echolucent medial layer as previously described. ${ }^{10-13,15,16,18}$ All segments were analyzed at $1-\mathrm{mm}$ intervals along the length of the arteries. Percent degrees atheroma (\% circumferential wall coverage) was calculated and compared between groups.

\section{Platelet aggregation}

Whole blood (60-70 mL) was collected from large bore central catheters into vacutainers containing $3.2 \%$ citrate prior to administration of heparin or contrast material and was used immediately for platelet experiments. Platelet-rich plasma (PRP) was obtained from citrate blood after centrifugation at $120 \mathrm{~g}$ for 5 minutes. After recovering the PRP, the blood samples were subjected to further centrifugation at $850 \mathrm{~g}$ 


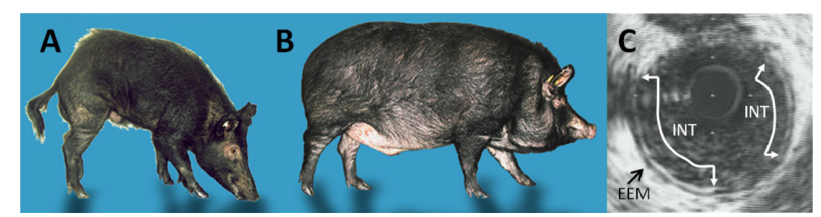

Figure I Ossabaw swine phenotypes and coronary atherosclerosis. Lean (A) and obese, metabolic syndrome (B) swine; (C) Coronary atheroma visualized in an animal with metabolic syndrome by intravascular ultrasound with arrows indicating neointimal border (INT) and external elastic membrane (EEM).

for 10 minutes to recover platelet-poor plasma (PPP). The resulting PRP and PPP were kept at room temperature for use within 1 hour. Platelets were stimulated at $37^{\circ} \mathrm{C}$ with adenosine diphosphate (ADP) at concentrations of $0.5,1$, $2.5,5,10$, and $20 \mu \mathrm{M}$, collagen at $1 \mu \mathrm{g} / \mathrm{mL}$ and arachidonic acid (AA) at $0.5 \mathrm{mM}$ (Chrono-log Corporation, Havertown, PA) as previously described. ${ }^{23}$ Thrombin was chosen over thrombin receptor agonist peptide (TRAP) as agonist due to inability of TRAP to induce consistent aggregation of porcine platelets by activation of platelet protease activated receptor-1 (PAR1). ${ }^{24}$

Platelets for thrombin experiments were washed by further centrifugation of PRP at $850 \mathrm{~g}$ for 10 minutes to remove plasma and reduce fibrin formation induced by thrombin during platelet aggregation. The supernatant was discarded and the remaining pellet resuspended in HEPES buffer (Sigma-Aldrich, St. Louis, MO) at a platelet concentration of $100 \times 10^{6} / \mathrm{mL}$. Aggregation of washed platelet suspension was measured after stimulation with thrombin (Chrono-log Corporation) at concentrations of 0.01, 0.05, 0.1 , and $0.5 \mathrm{U} / \mathrm{mL}$.

Aggregation measurements were performed with a Chronolog Lumi-Aggregometer (model-700; Chrono-log Corporation) with the AggroLink software package as described previously. ${ }^{23}$ Platelet aggregation was expressed as the maximal percent change in light transmittance from baseline (MPA) or as AUC within 8 minutes, using animal specific PPP or HEPES buffer as a reference.

Dose response curves and half maximal effective concentrations (EC50) were calculated for each animal using single ligand regression analysis for the different agonist concentrations of thrombin and ADP.

\section{Statistical analysis}

All statistical analyses were performed using Sigma Plot (version 11; Systat Software, San Jose, CA) and SPSS statistics (version 18; SPSS Inc., Somers, NY). As appropriate, $t$-tests were used to compare platelet aggregation between groups and means of EC50, with $P<0.05$ considered significant. Wilcoxon rank-sum test was used to compare nonparametric data. Single ligand regression analysis was performed to calculate EC50 for ADP and thrombin. Unless specified otherwise, values are represented as means \pm standard deviation (SD).

\section{Results \\ Metabolic syndrome characteristics}

Swine fed high calorie, high fat/cholesterol/fructose atherogenic diet were considerably more obese than those fed standard chow $(107.5 \pm 8.7$ vs $65.8 \pm 6.7 \mathrm{~kg}, P<0.01)$ and exhibited all the features of MetS compared with lean controls (Figure 1, Table 1).

Both systolic and diastolic hypertension were present in MetS pigs compared with lean controls (148/95 $\mathrm{mmHg}$ vs $126 / 80 \mathrm{mmHg}, P<0.01)$. Total cholesterol was almost 4-fold higher and triglycerides were roughly 2 -fold increased in obese pigs compared with lean controls. Obese pigs with

Table I Metabolic syndrome characteristics: animal body weight, systolic and diastolic blood pressure measurements, lipid profiles, insulin area under the curve (AUC) concentration during intravenous glucose tolerance test and percentage of circumferential atherosclerosis coverage on intravascular ultrasound evaluation of left anterior descending and circumflex arteries (mean \pm SD)

\begin{tabular}{|c|c|c|c|}
\hline & Metabolic syndrome $(n=10)$ & Lean $(n=10)$ & $P$ value \\
\hline Body weight (kg) & $107.5 \pm 8.7$ & $65.8 \pm 6.7$ & $<0.01$ \\
\hline Systolic blood pressure $(\mathrm{mmHg})$ & $148 \pm 1$ & $126 \pm 2$ & $<0.01$ \\
\hline Diastolic blood pressure $(\mathrm{mmHg})$ & $95 \pm 1$ & $80 \pm 2$ & $<0.01$ \\
\hline Cholesterol (mg/dL) & $220 \pm 174$ & $60.9 \pm 9$ & 0.025 \\
\hline Triglycerides (mg/dL) & $56 \pm 16$ & $26 \pm 8$ & $<0.01$ \\
\hline LDL (mg/dL) & $161 \pm 152$ & $17.4 \pm 9$ & 0.016 \\
\hline $\mathrm{HDL}(\mathrm{mg} / \mathrm{dL})$ & $36 \pm 11$ & $38 \pm 7$ & 0.68 \\
\hline LDL/HDL ratio & $3 \pm 1.2$ & $0.5 \pm 0.3$ & $<0.01$ \\
\hline Insulin AUC ( $\mu \mathrm{U} / \mathrm{mL})$ & 8596 & 3643 & $<0.00001$ \\
\hline $\begin{array}{l}\text { Circumferential coronary } \\
\text { atherosclerosis coverage (\%) }\end{array}$ & $33 \pm 2 \%$ & $25 \pm 2 \%$ & 0.013 \\
\hline
\end{tabular}

Abbreviations: HDL, high-density lipoprotein cholesterol; LDL, low-density lipoprotein cholesterol; SD, standard deviation. 

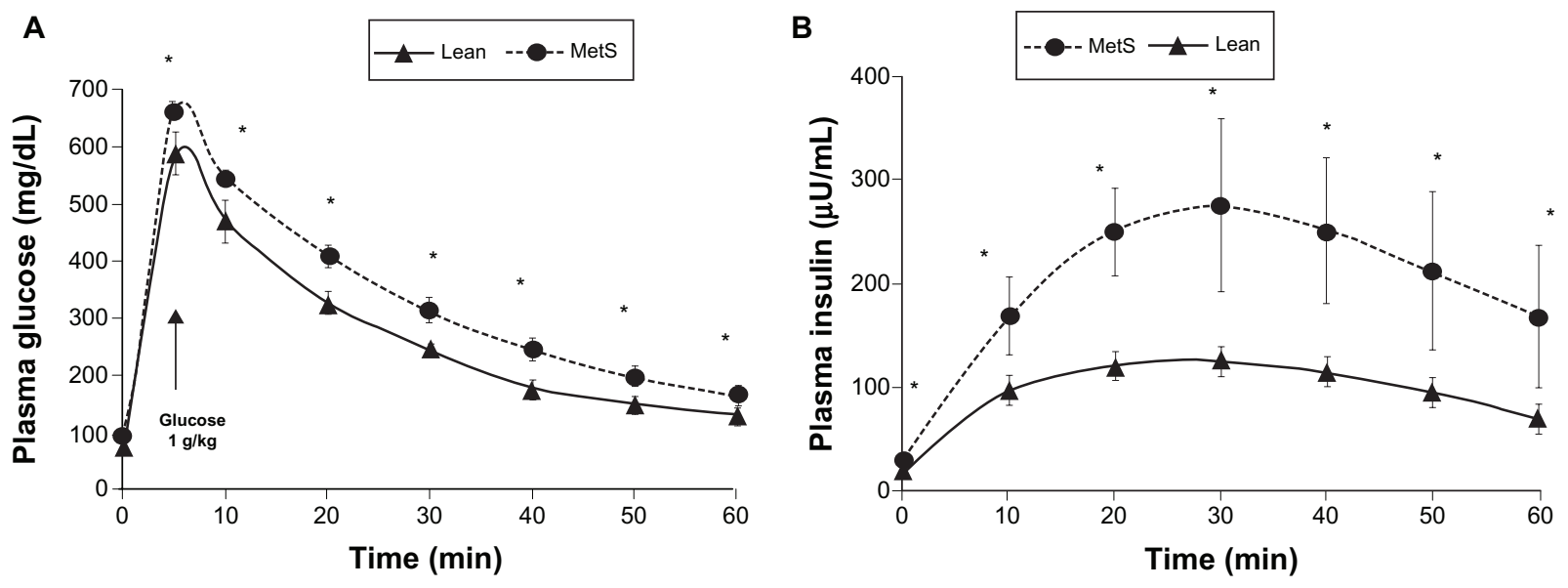

Figure 2 Intravenous glucose tolerance test: glucose plasma (A) and insulin plasma measurements $(\mathbf{B})$ at baseline and after intravenous administration of I g glucose/kg body weight in metabolic syndrome (MetS) vs Lean; ${ }^{*} P<0.05$.

MetS showed an almost 10-fold increase in LDL levels compared with lean controls with similar concentrations of HDL (Table 1).

MetS pigs demonstrated insulin resistance as evidenced by higher insulin concentrations and higher glucose concentrations following IVGTT (Table 1, Figure 2). It is noteworthy that animals with MetS exhibited significantly higher levels of insulin than leans even before administration of intravenous glucose bolus.

Intravascular ultrasound showed larger coronary atheroma volume as defined by percent circumferential wall coverage in obese pigs with MetS compared with lean controls ( $33 \pm 2 \%$ vs $25 \pm 2 \%, P=0.013$ ). An example of a coronary atheroma from a MetS animal is depicted in Figure 1.

\section{Platelet aggregation}

Platelet aggregation results for increasing doses of ADP (Figure 3) and for thrombin as agonists (Figure 4) are shown.
MetS animals showed a trend towards higher maximal platelet aggregation at submaximal concentrations of ADP, compared with leans with similar aggregation at maximal concentrations of ADP. For both ADP and thrombin EC50 were calculated for each sample using single ligand regression analysis. Overall, platelets from pigs with MetS were more sensitive to aggregation by ADP than platelets from lean controls with lower EC50 concentration (EC50 [MPA]: $1.83 \pm 1.3 \mu \mathrm{M}$ vs $3.64 \pm 2.2 \mu \mathrm{M} ; P=0.02$ ) (Figure 5). Higher velocity of platelet aggregation as defined by the slope of initial aggregation was measured for submaximal concentrations of ADP in animals with MetS vs lean controls with similar velocity for maximal concentrations of ADP (EC50 [slope]: $1.06 \pm 0.9$ $\mu \mathrm{M}$ vs $2.42 \pm 1.6 \mu \mathrm{M} ; P=0.02$ ) (Figures 3,5 ). There was a nonsignificant trend towards lower EC50: $(0.27 \pm 0.3$ vs $0.36 \pm 0.6 \mathrm{U} / 1 ; P=0.36$ ) (Figure 5) for aggregation induced by thrombin in platelets from MetS pigs compared with lean controls. No significant difference was found for slope of
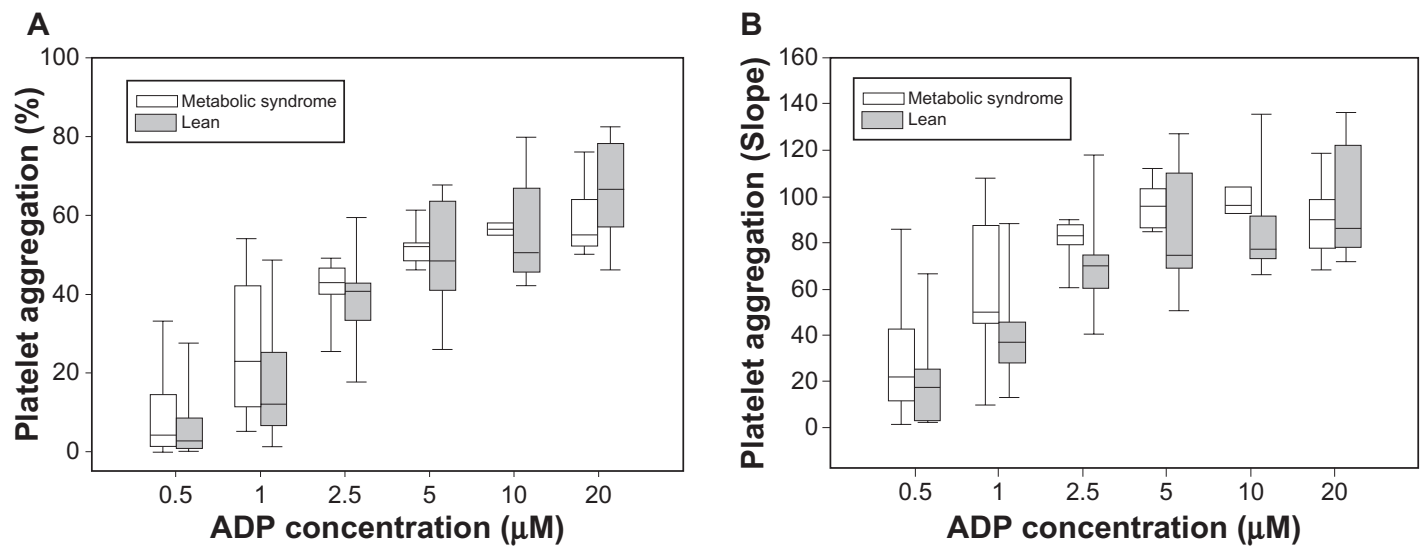

Figure 3 Platelet aggregation by adenosine diphosphate (ADP): maximal platelet aggregation (A) and slope (B) in response to increasing concentrations of ADP. 
A

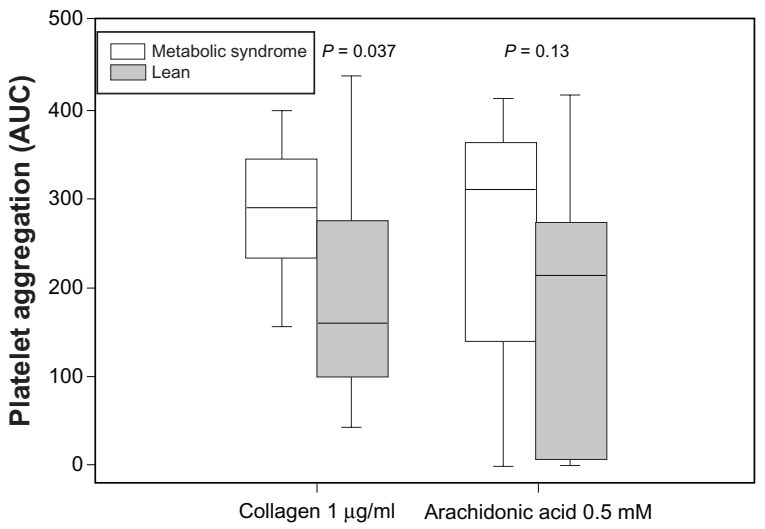

B

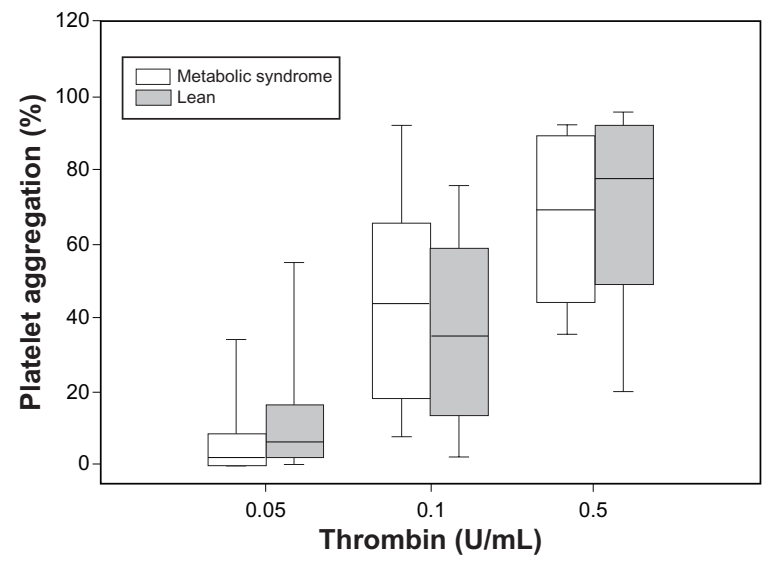

Figure 4 Platelet aggregation by collagen, arachidonic acid and thrombin: percent maximal platelet aggregation in response to collagen and arachidonic acid (A) and thrombin (B).

thrombin-induced platelet aggregation between MetS and lean controls (data not shown). Obese pigs with MetS demonstrated higher platelet aggregation in response to collagen than lean pigs (AUC: $286 \pm 74$ vs $198 \pm 123 ; P=0.037$ ) and a trend towards higher platelet aggregation in response to AA (AUC: $260 \pm 151$ vs $178 \pm 145 ; P=0.13$ ) (Figure 4).

\section{Discussion}

Obesity and MetS are important cardiovascular risk factors involved in the development of atherosclerotic disease. It is well established that insulin resistance and the emergence of diabetes mellitus are associated not only with atherosclerotic plaque formation and progression, but also influence the thrombotic phenotype of affected individuals. ${ }^{1,5-9}$ Numerous studies have documented that patients with diabetes mellitus exhibit higher levels of activated platelets and are more likely to show inadequate inhibition of platelet aggregation by aspirin or thienopyridines. ${ }^{25-27}$ As platelets become activated they form aggregates with leukocytes through binding by p-selectin and experience cross talk with leukocytes that leads to secretion of tissue factor by leukocytes and generation of thrombin. ${ }^{28,29}$ Platelet-leukocyte aggregates that are increased in patients with diabetes mellitus promote rolling and delivery of leukocytes to the endothelium and promote transmigration of leukocytes, and may thus promote vascular inflammation and atherosclerosis formation. ${ }^{1,8,28,29}$ Consistent with these ex vivo findings, patients with coronary disease and diabetes mellitus are at highest risk for recurrent myocardial infarction and stent thrombosis after percutaneous coronary interventions and more likely to be clopidogrel 'nonresponders' than nondiabetic patients. ${ }^{30}$

Several mechanisms have been postulated to play a role in increasing platelet reactivity associated with MetS, including direct effects of free fatty acids, adiponectin, and leptin on platelets. ${ }^{1,31,32}$ A recent study examining the effect of weight loss suggested altered platelet inhibitory effects of nitric oxide and prostacyclin in obese individuals. ${ }^{33}$ Also, previous studies have suggested that insulin resistance may lead to a loss of insulin-mediated inhibition of P2Y12 receptor signaling, thereby increasing ADP-mediated platelet aggregation. ${ }^{34}$
A

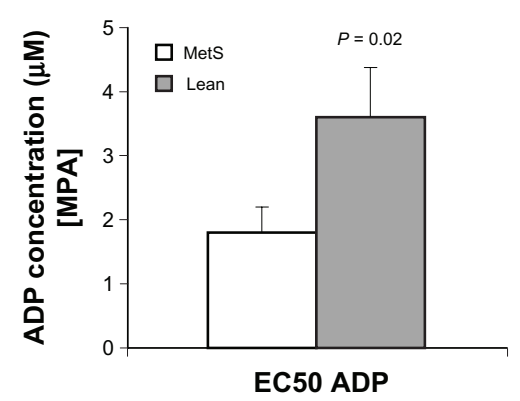

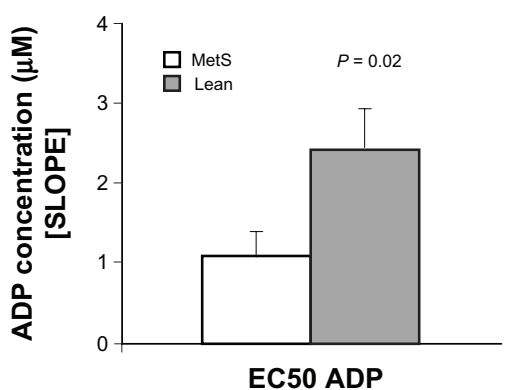

C

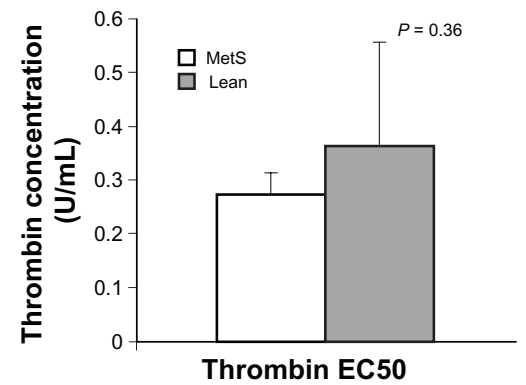

Figure 5 Half maximal effective concentration (EC50) concentrations adenosine diphosphate (ADP) (maximal platelet aggregation [MPA] and slope) and thrombin. EC50 for ADP-induced MPA (A), ADP slope (B), and thrombin-induced MPA (C) (mean \pm SE).

Abberviations: MetS, metabolic syndriog. 
Platelets from obese Ossabaw swine with MetS were more sensitive to lower doses of ADP as defined by a lower EC50 concentration and showed evidence of faster initial platelet aggregation in response to submaximal concentrations of ADP. Similarly, higher platelet aggregation was shown in platelets from MetS pigs compared with platelets from lean controls when using a fixed concentration of collagen and AA as agonist. The molecular mechanisms responsible for this effect remain unclear. It can be deduced, however, that platelets from animals with MetS may have a higher tendency for aggregation under shear conditions than platelets from animals without MetS.

Although numerous rodent and small animal models are available for atherosclerosis research, we still lack large animal models that allow study of the progression of atherosclerosis and use of experimental devices in human-sized animals. ${ }^{35}$ Other miniature swine models of MetS are limited by lack of hypertension and also coronary atherosclerosis has not been documented. ${ }^{36}$ The Ossabaw swine model has appeared as a viable option for the study of coronary interventional devices and coronary disease in an animal that is close to human size and exhibits the same metabolic changes that are associated with vascular disease in the human population. ${ }^{10-18}$ Within our study Ossabaw swine who were fed excess calorie atherogenic diet for 44 weeks developed all the features of MetS compared with a lean control group, including central abdominal obesity, systolic and diastolic hypertension, hypertriglyceridemia, hypercholesteremia, insulin resistance, impaired glucose tolerance, and coronary atherosclerosis.

The effect of type 1 diabetes mellitus on platelet aggregation has been studied in swine models. ${ }^{37}$ This is the first study to examine the platelet aggregatory phenotype of obese Ossabaw swine with MetS and insulin resistance compared with non-MetS pigs. Shukla et al included an atherogenic diet fed group of Sinclair miniature swine, who failed to show increased platelet reactivity, although that group was dyslipidemic, similar to the MetS Ossabaw swine in the current study. ${ }^{37}$ Thus, the increased sensitivity of MetS Ossabaw platelets to ADP and collagen may potentially be due to other factors in MetS, such as insulin resistance. Clearly, this is a highly distinguishing feature of Ossabaw miniature swine compared with Sinclair or Yucatan swine, which do not show primary insulin resistance when fed excess calorie diet. ${ }^{20,35}$

\section{Conclusions}

This study adds additional information to the phenotypic characterization of the obese Ossabaw swine and suggests the presence of a pro-aggregatory environment in Ossabaw swine with MetS that may be comparable to humans with MetS. Combined with the finding of spontaneous coronary atherosclerosis in obese Ossabaw swine, these data support the use of this model to study intravascular devices in a metabolic environment comparable to human patients with MetS. The obese Ossabaw swine may be an ideal large animal model for the study of the effects of high caloric-high fat diet on the genesis of MetS and may be useful to further examine the mechanisms associated with increased platelet reactivity in humans with MetS.

\section{Acknowledgment}

The study was supported by NIH RR013223 \& Eli Lilly and Co. grants to M Sturek and NIH/NCRR RR025761 to R Kreutz.

\section{Disclosure}

The authors report no conflicts of interest.

\section{References}

1. Alessi MC, Juhan-Vague I. Metabolic syndrome, haemostasis and thrombosis. Thromb Haemost. 2008;99(6):995-1000.

2. Ford ES. Risks for all-cause mortality, cardiovascular disease, and diabetes associated with the metabolic syndrome: a summary of the evidence. Diabetes Care. 2005;28(7):1769-1778.

3. Stern MP, Williams K, Gonzalez-Villalpando C, Hunt KJ, Haffner SM. Does the metabolic syndrome improve identification of individuals at risk of type 2 diabetes and/or cardiovascular disease? Diabetes Care. 2004;27(11):2676-2681.

4. Ford ES, Giles WH, Dietz WH. Prevalence of the metabolic syndrome among US adults: findings from the third National Health and Nutrition Examination Survey. JAMA. 2002;287(3):356-359.

5. Meerarani P, Badimon JJ, Zias E, Fuster V, Moreno PR. Metabolic syndrome and diabetic atherothrombosis: implications in vascular complications. Curr Mol Med. 2006;6(5):501-514.

6. Kim JA, Montagnani M, Koh KK, Quon MJ. Reciprocal relationships between insulin resistance and endothelial dysfunction: molecular and pathophysiological mechanisms. Circulation. 2006;113(15):1888-1904.

7. Basili S, Pacini G, Guagnano MT, et al. Insulin resistance as a determinant of platelet activation in obese women. $\mathrm{J} \mathrm{Am} \mathrm{Coll} \mathrm{Cardiol.}$ 2006;48(12):2531-2538.

8. Arteaga RB, Chirinos JA, Soriano AO, et al. Endothelial microparticles and platelet and leukocyte activation in patients with the metabolic syndrome. Am J Cardiol. 2006;98(1):70-74.

9. Schneider DJ, Hardison RM, Lopes N, Sobel BE, Brooks MM; Pro-Thrombosis Ancillary Study Group. Association between increased platelet P-selectin expression and obesity in patients with type 2 diabetes: a BARI 2D (Bypass Angioplasty Revascularization Investigation 2 Diabetes) substudy. Diabetes Care. 2009;32(5):944-949.

10. Sturek M, Alloosh M, Wenzel J, et al. Ossabaw Island miniature swine: cardiometabolic syndrome assessment. In: Swindle MM, editor. Swine in the Laboratory: Surgery, Anesthesia, Imaging, and Experimental Techniques. Boca Raton, FL: CRC Press; 2007:397-402.

11. Dyson MC, Alloosh M, Vuchetich JP, Mokelke EA, Sturek M. Components of metabolic syndrome and coronary artery disease in female Ossabaw swine fed excess atherogenic diet. Comp Med. 2006;56(1):35-45. 
12. Lloyd PG, Sheehy AF, Edwards JM, Mokelke EA, Sturek M. Leukemia inhibitory factor is upregulated in coronary arteries of Ossabaw miniature swine after stent placement. Coron Artery Dis. 2008;19(4):217-226.

13. Edwards JM, Alloosh M, Long X, et al. Adenosine A receptors in neointimal hyperplasia and in-stent stenosis in Ossabaw miniature swine. Coron Artery Dis. 2008;19(1):27-31.

14. Bratz IN, Dick GM, Tune JD, et al. Impaired capsaicin-induced relaxation of coronary arteries in a porcine model of the metabolic syndrome. Am J Physiol Heart Circ Physiol. 2008;294(6):H2489-H2496.

15. Edwards JM, Neeb ZP, Alloosh MA, et al. Exercise training decreases store-operated $\mathrm{Ca}^{2+}$ entry associated with metabolic syndrome and coronary atherosclerosis. Cardiovasc Res. 2010;85(3):631-640.

16. Bender SB, Tune JD, Borbouse L, Long X, Sturek M, Laughlin MH. Altered mechanism of adenosine-induced coronary arteriolar dilation in early-stage metabolic syndrome. Exp Biol Med. 2009; 234(6):683-692.

17. Lee L, Alloosh M, Saxena R, et al. Nutritional model of steatohepatitis and metabolic syndrome in the Ossabaw miniature swine. Hepatology. 2009;50(1):56-67.

18. Borbouse L, Dick GM, Asano S, et al. Impaired function of coronary $\mathrm{BK}_{\mathrm{Ca}}$ channels in metabolic syndrome. Am J Physiol Heart Circ Physiol. 2009;297(5):H1629-H1637.

19. Bell LN, Lee L, Saxena R, et al. Serum proteomic analysis of diet-induced steatohepatitis and metabolic syndrome in the Ossabaw miniature swine. Am J Physiol Gastrointest Liver Physiol. 2010;298(5):G746-G754.

20. Neeb ZP, Edwards JM, Alloosh M, Long X, Mokelke EA, Sturek M. Metabolic syndrome and coronary artery disease in Ossabaw compared with Yucatan swine. Comp Med. 2010;60(4):300-315.

21. National Research Council. Guide for the Care and Use of Laboratory Animals. Washington, DC: The National Academies Press; 1996.

22. Beaver BV, Reed W, Leary S, et al. 2000 Report of the AVMA panel on euthanasia. J Am Vet Med Assoc. 2001;218(5):669-696.

23. Kreutz RP, Tantry US, Bliden KP, Gurbel PA. Inflammatory changes during the 'common cold' are associated with platelet activation and increased reactivity of platelets to agonists. Blood Coagul Fibrinolysis. 2007;18(8):713-718.

24. Kinlough-Rathbone RL, Rand ML, Packham MA. Rabbit and rat platelets do not respond to thrombin receptor peptides that activate human platelets. Blood. 1993;82(1):103-106.
25. Franchini M, Targher G, Montagnana M, Lippi G. The metabolic syndrome and the risk of arterial and venous thrombosis. Thromb Res. 2008;122(6):727-735.

26. Price MJ, Nayak KR, Barker CM, Kandzari DE, Teirstein PS. Predictors of heightened platelet reactivity despite dual-antiplatelet therapy in patients undergoing percutaneous coronary intervention. Am J Cardiol. 2009;103(10):1339-1343.

27. Vaduganathan M, Alviar CL, Arikan ME, et al. Platelet reactivity and response to aspirin in subjects with the metabolic syndrome. Am Heart J. 2008;156(5):1002.e1-1002.e7.

28. Massberg S, Brand K, Grüner S, et al. A critical role of platelet adhesion in the initiation of atherosclerotic lesion formation. J Exp Med. 2002;196(7):887-896.

29. Wagner DD. New links between inflammation and thrombosis Arterioscler Thromb Vasc Biol. 2005;25(7):1321-1324.

30. Holmes DR Jr, Kereiakes DJ, Garg S, et al. Stent thrombosis. J Am Coll Cardiol. 2010;56(17):1357-1365.

31. Kato H, Kashiwagi H, Shiraga M, et al. Adiponectin acts as an endogenous antithrombotic factor. Arterioscler Thromb Vasc Biol. 2006;26(1):224-230.

32. Konstantinides S, Schäfer K, Koschnick S, Loskutoff DJ. Leptindependent platelet aggregation and arterial thrombosis suggests a mechanism for atherothrombotic disease in obesity. $J$ Clin Invest. 2001;108(10):1533-1540.

33. Russo I, Traversa M, Bonomo K, et al. In central obesity, weight loss restores platelet sensitivity to nitric oxide and prostacyclin. Obesity. 2010;18(4):788-797.

34. Ferreira IA, Mocking AI, Feijge MA, et al. Platelet inhibition by insulin is absent in type 2 diabetes mellitus. Arterioscler Thromb Vasc Biol. 2006;26(2):417-422.

35. Otis CR, Wamhoff BR, Sturek M. Hyperglycemia-induced insulin resistance in diabetic dyslipidemic Yucatan swine. Comp Med. 2003;53(1):53-64.

36. Christoffersen BO, Grand N, Golozoubova V, Svendsen O, Raun K. Gender-associated differences in metabolic syndrome-related parameters in Göttingen minipigs. Comp Med. 2007;57(5):493-504.

37. Shukla SD, Kansra SV, Reddy MA, Shukla SM, Klachko DM, Sturek M. Platelets from diabetic pigs exhibit hypersensitivity to thrombin. Comp Med. 2008;58(5):481-484.

\section{Publish your work in this journal}

Diabetes, Metabolic Syndrome and Obesity: Targets and Therapy is an international, peer-reviewed open-access journal committed to the rapid publication of the latest laboratory and clinical findings in the fields of diabetes, metabolic syndrome and obesity research Original research, review, case reports, hypothesis formation, expert opinion and commentaries are all considered for publication. The manuscript management system is completely online and includes a very quick and fair peer-review system, which is all easy to use. Visit http://www.dovepress.com/testimonials.php to read real quotes from published authors. 